Quenching of the nonlocal electron heat transport by large external magnetic fields in a laser produced plasma measured with imaging Thomson scattering

D. H. Froula, P. Davis, B. B. Pollock, L. Divol, J. S. Ross, J. Edwards, R. Town, D. Price, S. H. Glenzer, A. A. Offenberger, G. R. Tynan, A. N. James

May 3, 2006

Physical Review Letters 
This document was prepared as an account of work sponsored by an agency of the United States Government. Neither the United States Government nor the University of California nor any of their employees, makes any warranty, express or implied, or assumes any legal liability or responsibility for the accuracy, completeness, or usefulness of any information, apparatus, product, or process disclosed, or represents that its use would not infringe privately owned rights. Reference herein to any specific commercial product, process, or service by trade name, trademark, manufacturer, or otherwise, does not necessarily constitute or imply its endorsement, recommendation, or favoring by the United States Government or the University of California. The views and opinions of authors expressed herein do not necessarily state or reflect those of the United States Government or the University of California, and shall not be used for advertising or product endorsement purposes. 


\title{
Quenching of the nonlocal electron heat transport by large external magnetic fields in a laser produced plasma measured with imaging Thomson scattering
}

\author{
D. H. Froula, ${ }^{*}$ P. Davis ${ }^{\dagger}$ B. B. Pollock, ${ }^{\ddagger}$ L. Divol, J. S. Ross, J. Edwards, R. Town, D. Price, and S. H. Glenzer \\ L-399, Lawrence Livermore National Laboratory, P.O. Box 808, Livermore, CA 94551, USA
}

\author{
A. A. Offenberger \\ Department of Electrical Engineering, University of Alberta, Edmonton, Alberta, Canada T6G 2V4
}

G. R. Tynan and A. N. James

Engineering Department, University of California at San Diego

(Dated: April 7, 2006)

\begin{abstract}
We present a direct measurement of the quenching of nonlocal heat transport in a laser produced plasma by high external magnetic fields. Temporally resolved measurements of the electron temperature profile transverse to a high power laser beam were obtained using imaging Thomson scattering. The results are simulated with the $2 \mathrm{D}$ hydrodynamic code LASNEX with a recently included magnetic field model that self-consistently evolves the fields in the plasma.
\end{abstract}

PACS numbers: 52.25.Os, 52.35.Fp, 52.50.Jm

Keywords: ion-acoustic wave, magnetic fields, Thomson scattering

Inertial confinement fusion and high energy density science experiments require a detailed understanding of heat transport in the presence of magnetic fields [1-3]. The indirect drive approach to inertial confinement fusion employs high-Z radiation cavities (hohlraums) filled with a low density gas to convert laser energy to soft x-ray radiation that drives a fusion capsule implosion by ablation pressure [4]. To achieve the desired implosion symmetry, the laser energy deposition and transport must be correctly modeled in the presence of magnetic fields generated by the laser beams [5]. Cross-magnetic-field heat transport is also important for understanding magnetic fusion plasmas, astrophysical observations [6], and basic plasma physics [7].

Current hydrodynamic simulations used to determine ignition design parameters employ a modified transport model that calculates heat flux using the minimum calculated value from either classical Spitzer-Harm or freestreaming (limited by an arbitrary factor) [8]. The fluxlimiter was introduced primarily to reproduce experiments where simulations involve energy transport from the laser absorption region into a solid target [9]. However, the coronal plasmas have been difficult to model, though Spitzer-Harm diffusion has been successfully applied in a few experiments $[10,11]$. Currently there are outstanding questions regarding the relative importance of nonlocal heat transport and the effects of self generated magnetic fields in the complex hohlraum plasmas; furthermore, there are no measurements of electron heat transport in the presence of magnetic fields to compare with modeling calculations.

In this letter, we present the first observation of the localization of the electron heat transport by high magnetic fields in high temperature laser produced plasma. It is found that a 12 Tesla external magnetic field ori-

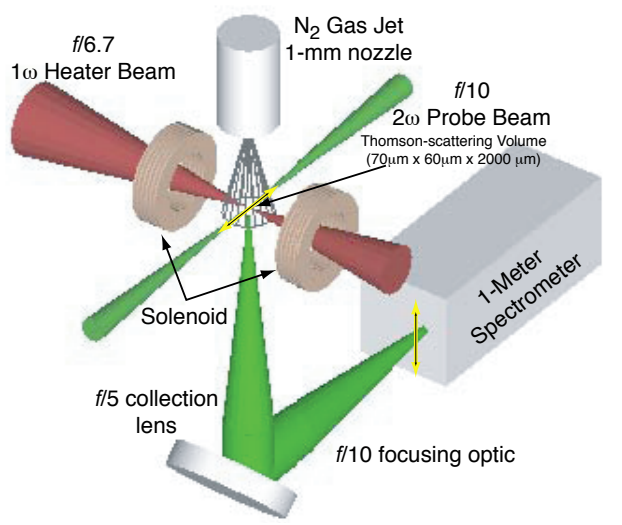

FIG. 1: The experimental setup is shown where a $1 \omega$ high power (1-ns square) laser beam initially heats a narrow $200 \mu \mathrm{m}$ plasma channel in $\mathrm{N}_{2}$ gas injected by a $1.5 \mathrm{~mm}$ nozzle. Two solenoids produce a uniform high magnetic field $(B=13$ Tesla) parallel to the heater beam. A collective Thomsonscattering diagnostic measures the electron temperature profile perpendicular to the heater beam; a spectrometer slit is imaged perpendicular to the heater beam to collect light scattered from a low energy $2 \omega$ probe laser.

ented parallel to a high power laser beam inhibits electrons from propagating perpendicular to the axis of the heater beam; the magnetic field reduces the heat flux by a factor of 15 . With this reduction in heat flux, the plasma expands adiabatically. The magnetic Reynold's number is large therefore, the magnetic field is frozen into the plasma and as the plasma expands $(\beta=$ plasma $/$ magnetic pressure $=20)$, the magnetic field follows the density and is compressed at the heat front. Spatially and temporally resolved temperature measurements using imaging Thomson scattering provides data for direct comparison with hydrodynamic sim- 
ulations that include magnetic fields. The Larmor radius $\left(r_{c}=m v / e B=5 \mu \mathrm{m}\right)$ is much smaller than the temperature scale length, thereby localizing the electron flux $\left(\mathrm{r}_{c}<<\nabla T_{e} / T_{e}\right)$ and classical Braginskii heat transport is successfully used to model the heat wave propagation.

Nonlocal transport effects $\left(\mathrm{v}_{e} \tau_{e i}>\nabla T_{e} / T_{e}\right)$ on heat wave propagation have been observed in similar experiments without magnetic fields [12]. In our experiment we reproduce the nonlocal transport effects without an external magnetic field and show that a 12 Tesla magnetic field is sufficient to localize the electron heat flux $\left(\mathrm{v}_{e} \tau_{c}<\right.$ $\left.\nabla T_{e} / T_{e}\right)$. These experimental results compare well to hydrodynamic simulations using a local heat transport model that includes magnetic fields [13]. The measurements are well reproduced without invoking "turbulent" transport models $\left(\mathrm{r}_{c}>>\lambda_{D e}\right)$ which is required in most MHD research due to non-thermal fluctuations such as unstable waves or broad band turbulence [14].

This experiment was performed in the Janus Laser Facility at the Lawrence Livermore National Laboratory (Fig. 1). Plasmas were produced in a nitrogen gas jet with a $1.5 \mathrm{~mm}$ diameter cylindrical nozzle and an operating upstream pressure of 100 psi. The opening of the nozzle was aligned $1.5 \mathrm{~mm}$ from the target chamber center (TCC). The neutral gas density was well characterized using interferometery [15]; the peak electron density for our conditions (fully ionized nitrogen) was $\mathrm{n}_{e}=1.5 \times 10^{19} \mathrm{~cm}^{-3}$. A $1 \omega(1054 \mathrm{~nm}), 1 \mathrm{~ns}$ square, $100 \mathrm{~J}$ laser beam was focused with a 1 meter focal length lens (f/6.7) through a continuous phase plate producing a $150 \mu \mathrm{m}$ diameter focus at TCC.

A second laser beam frequency doubled $(2 \omega)$ to produce $0.5 \mathrm{~J}$ at 200 ps was used to probe the plasma perpendicular to the high power beam by focusing the laser light with a $40 \mathrm{~cm}$ focal length lens (f/10) to a $70 \mu \mathrm{m}$ diameter spot at TCC. The light Thomson scattered from the probe beam, perpendicular to the beam plane, was collected and collimated by an f/ 5 lens and imaged with a f/15 focusing lens on to the $200 \mu \mathrm{m}$ slit of a 1 meter, 2400 groves/mm imaging spectrometer. The Thomsonscattering collection system had a magnification of 3 . An intensified gated CCD camera with $26 \mu \mathrm{m}$ square pixels was coupled to the spectrometer providing a spectral resolution of $0.089 \mathrm{~nm}$ and a spatial resolution perpendicular to the high power beam better than 50 microns.

The Thomson-scattering volume was defined by the beam waist of the probe laser $(70 \mu \mathrm{m})$ and the projection of the spectral slit into the plasma plane $(60 \mu \mathrm{m})$. The time between the beams was varied to study the temporal evolution of the plasma heating; the relative beam timing was known to $200 \mathrm{ps}$ and the jitter between the beams was 100 ps.

An external magnetic field parallel to the heater beam was generated by driving high currents through a modified Bitter magnet [16]. The magnet was driven with a pulse power system capable of delivering a peak current

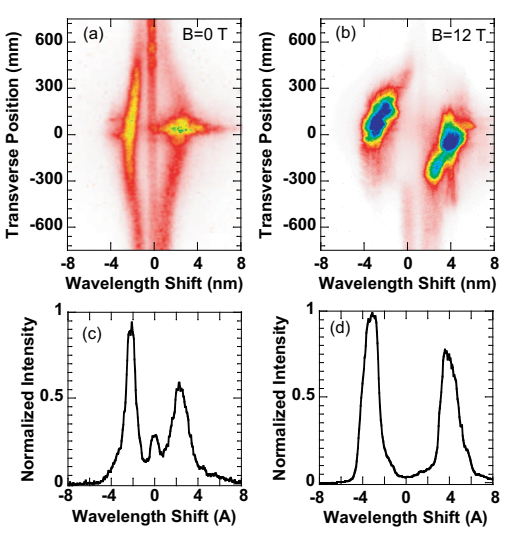

FIG. 2: Spatially resolved Thomson-scattering spectra are presented for conditions: (a) without an applied magnetic field $(\mathrm{B}=0 \mathrm{~T})$ near the end of the heating pulse and, (b) with a high magnetic field $(B=12 \mathrm{~T}), 500$ ps after the end of the heater; the magnetic field both reduces the extent of the plasma and increases the electron temperature by more than a factor of 3 . The spectra near the center of the heated region are shown for both conditions (c) $\mathrm{B}=0 \mathrm{~T},(\mathrm{~d}) \mathrm{B}=12 \mathrm{~T}$; data are fit with a form factor to determine the electron temperatures from the separation between the spectral features.

of $15 \mathrm{kA}$, resulting in a $12 \mathrm{~T}$ field in vacuum at the TCC. The magnetic field was shown to be constant over the laser experiment scale lengths ( $\sim 1 \mathrm{~mm}, 1 \mathrm{~ns})$ [17].

Figure 2 compares the Thomson-scattering spectra for $\mathrm{B}=0 \mathrm{~T}$ and $\mathrm{B}=12 \mathrm{~T}$; the separation between the spectral features is a direct measure of the electron temperature. From Fig. 2, the transverse heat front has clearly been inhibited and the separation between the features inside of the heated region is significantly larger when an external magnetic field of $12 \mathrm{~T}$ is applied. The electron temperature profile is extracted by fitting the measured frequency spectrum every 50 microns with the frequency-dependent Thomson-scattering form factor $\mathrm{S}(\mathrm{k}, \omega)$ [18]. The average ionization state is calculated using a Thomas-Fermi model [19] giving $\mathrm{Z}$ in the range of 4-7 for the electron temperature between $\mathrm{T}_{e}=20 \mathrm{eV}$ and $\mathrm{T}_{e}=350 \mathrm{eV}[12]$. This data and form-fitting illustrates the direct correlation between the Thomson-scattered spectral peaks and the electron temperature. Including errors in the determination of $\mathrm{Z}$, the absolute temperature is determined to within an accuracy of $20 \%$ and the relative temperature is known to better than $10 \%$ accuracy.

We speculate that the increased scattering signal observed around the center of the heater beam is a result of filamentation [20]. When the probe crosses the heater beam region it undergoes scattering from large amplitude long-scale length density fluctuations produced by the filamentation instability driven by the heater beam.

Figure 3 compares the electron temperature profiles with and without a magnetic field 500 ps after the rise of heater beam. The electron temperature profile for spectra taken when no external magnetic field is applied 


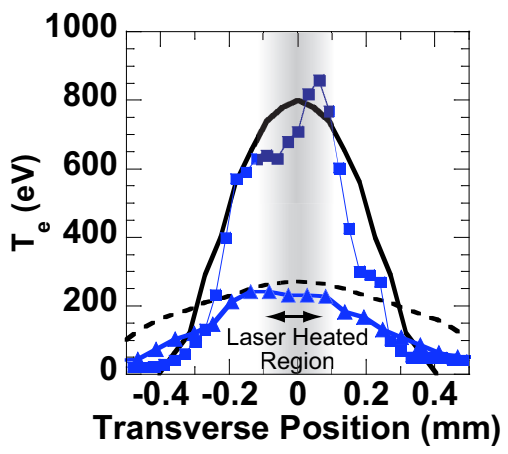

FIG. 3: The electron temperature profiles for no magnetic field (triangles) and an externally applied field of 12 Tesla (squares) show an increase in peak electron temperature and a corresponding reduction in the propagation of the heat wave perpendicular to the magnetic field. LASNEX simulations using a flux-limited diffusion model $(f=1)$ without magnetic fields (dashed line) under estimate the extent of the heat wave consequently simulating a higher peak electron temperature while simulations using the magnetic field package (solid line) reproduce the experimental results.

$(\mathrm{B}=0 \mathrm{~T})$ shows the heat front extends out well past 500 microns from the center of the heater beam. This is in stark contrast to the profile when a high external magnetic field $(\mathrm{B}=12 \mathrm{~T})$ is applied; the heat front has propagated less than 350 microns. This is direct evidence for the reduction in electron heat flux by an external magnetic field. Furthermore, these results show the quenching of the nonlocal heat transport by externally applied magnetic fields.

The results presented in Figure 3 are compared to simulations using the hydrodynamic code LASNEX [8] used to solve the classical heat transport equations originally presented by Braginskii [21]. In our simulations the electron heat flux is determined by the minimum between the Braginskii heat flux and the free-streaming heat flux, $\mathrm{q}=\min \left(\mathrm{n}_{e} \mathrm{~T}_{e} \mathrm{v}_{e}, q_{B}\right)$;

$$
\mathbf{q}_{B}=-\kappa_{\|} \nabla_{\|} T_{e}-\kappa_{\perp} \nabla_{\perp} T_{e}-\kappa_{\wedge} \mathbf{b} \times \nabla_{\perp} T_{e}
$$

where $\mathbf{b}$ is a unit vector along the magnetic field and the conductivities can be approximated for the high magnetic field simulations by $[13,22]$,

$$
\kappa_{\|}=3.2 \frac{Z n_{e} T_{e} \tau_{e i}}{m_{e}} ; \kappa_{\perp}=4.7 \frac{Z n_{e} T_{e}}{m_{e} \omega_{c e}^{2} \tau_{e i}^{2}} ; \kappa_{\wedge}=2.5 \frac{Z n_{e} T_{e}}{m_{e} \omega_{c e}} ;
$$

For our axisymmetric geometry, $\mathbf{b} \times \nabla_{\perp} T_{e}=0$ and the perpendicular and parallel conductivities can be compared to estimate a reduction in heat flow of $\kappa_{\|} / \kappa_{\perp}=15$.

Our LASNEX simulations propagate a $100 \mathrm{~J}, 1 \mathrm{~ns}$ square pulse with a $350 \mathrm{ps}$ rise and fall measured to the half-intensity point into a constant two dimensional density slab $\left(1.5 \times 10^{19} \mathrm{~cm}^{-} 3\right)$. The external magnetic field is imposed around the beam $\left(\mathrm{B}_{\theta}\right.$-field) in order to take ad-

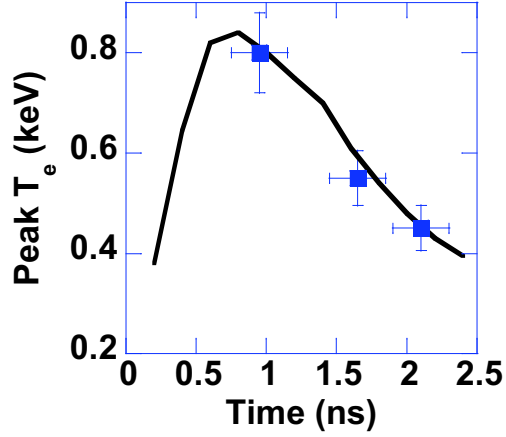

FIG. 4: The evolution of the measured peak electron temperature (squares) is in good agrement with hydrodynamic simulations that use a Braginskii heat transport model (line).

vantage of the ability of LASNEX to correctly propagate the evolution of the field.

For our conditions without magnetic fields, it has previously been shown that classical diffusion is not able to reproduce the heat wave propagation even when a flux limiter is used and that nonlocal transport effects play an important role in the determination of the plasma conditions [12]. Figure 3 reproduces these results showing that the Braginskii diffusion model (including self generated magnetic fields) does not correctly calculate the electron temperature profile when no external magnetic fields are present. The comparison shows that self generated magnetic fields do not account for the nonlocal nature of the heat flux; the gradient in the density is parallel to the gradient in electron temperature therefore, self generated fields are small.

Figure 4 shows the temporal evolution of the peak electron temperature when an external magnetic field of $\mathrm{B}=12$ Tesla is applied. Simulations using the magnetic field package in LASNEX reproduce this temperature evolution.

For our conditions, the magnetic field serves to restrict the electron thermal conduction losses but is not sufficient to confine the laser-heated core $(\beta=20)$. Initially the simulations show a background magnetic field through out the plasma. At $1.5 \mathrm{~ns}$, the field at the center of the plasma drops from the vacuum field of $12 \mathrm{~T}$ to $6 \mathrm{~T}$, while the field at the heat front is compressed to nearly $16 \mathrm{~T}$. This compression closely follows the radial density compression due to the expanding plasma; initially, the magnetic filed is trapped in the plasma, but as the temperature gradient resulting from the local laser beam heating creates a pressure gradient lowering the density in the center of the plasma, the field is pulled out by plasma expansion and compressed at the heat front.

The evolution of the magnetic filed can be derived from Ohm's law. The magnetic field moves with the flow and 
diffuses due to joule heating;

$$
\frac{\partial \mathrm{B}_{z}}{\partial t}-\nabla \cdot\left(\mathbf{v B}_{z}\right)=\frac{\eta}{\mu_{o}} \nabla^{2} \mathbf{B}
$$

where $\eta_{\perp} \sim 1 \times 10^{-4} Z \ln (\Lambda) / T_{e V}^{3 / 2}$ is the plasma resistivity and $\ln (\Lambda) \sim 8$ is the Coulomb logarithm. Equation 3 shows that when the plasma resistivity is small (i.e. perfect conductor), magnetic field transport by convection greatly exceeds transport by diffusion and the magnetic field will be frozen into the expansion so that [23],

$$
\frac{\mathrm{n}(\mathrm{t})}{\mathrm{n}(\mathrm{t}=0)} \sim \frac{\mathrm{B}(\mathrm{t})}{\mathrm{B}(\mathrm{t}=0)}
$$

Using the electron temperature $\left(\mathrm{T}_{e}=20 \mathrm{eV}\right)$ required to initially ionize the gas $(\mathrm{Z}=1)$ and the scale-length given by the transverse laser beam profile $(\mathrm{L} \sim 100 \mu \mathrm{m})$, the diffusion time during the heating is estimated to be $\tau_{d}=\mu_{o} L^{2} / \eta_{\perp}=5.6 \mathrm{~ns}$. Therefore, during plasma formation which takes place over the rise time of the laser pulse $\left(\tau_{L}=100 \mathrm{ps}\right)$, the magnetic field does not have time to diffuse $\left(\tau_{L} / \tau_{d}<<1\right)$ before the resistivity can be neglected due to the rapid increase in electron temperature; consequently the magnetic field is initially frozen into the plasma.

In summary, Thomson-scattering measurements have been successfully employed to demonstrate quenching of nonlocal heat transport in the presence of a large confining magnetic field. A 12 T external magnetic field parallel to a high power laser beam is shown to reduce the heat flux by a factor of 15 when compared with hydrodynamic simulations using a Braginskii heat transport model. The simulations using classical heat transport (i.e. no turbulence) in the presence of a magnetic field reproduce the measured heat wave propagation. The models tested in this study are currently being used to assess the importance of magnetic fields in predicting plasma conditions in inertial confinement fusion and high energy density science experiments.

We would like to acknowledge the efforts of the Janus Laser Crew. We thank G. Ullery, S. Fulkerson, J. Bower, and J. Satariano for their support in designing and building the pulse power system used to produce the high magnetic fields. This work was performed under the auspices of the U.S. Department of Energy by the Lawrence Livermore National Laboratory under Contract No. W7405-ENG-48.

* Electronic address: froula1@llnl.gov

$\dagger$ Also at Engineering Department, University of British Columbia.

¥ Also at Engineering Department, University of Pacific, 3601 Pacific Avenue, Stockton, CA 95211

[1] P. Nicolai et al., Phys. Plasmas 7, 4250 (2000).

[2] A. A. Offenberger and N. H. Burnett, Can. J. Phys. 53, 1360 (1975).

[3] M. G. Haines, Phys. Rev. Lett. 78, 254 (1997).

[4] J. D. Lindl et al., Phys. Plasmas 11, 339 (2004).

[5] S. H. Glenzer et al., Physical Review E 62 (2000).

[6] D. Ryutov et al., ASTROPHYS J 518, 821 (1999), part 1 Article 211KA English Times Cited:62 Cited References Count: 43

[7] C. Driscoll et al., Phys. Plasmas 9, 1905 (2002).

[8] G. B. Zimmerman and W. L. Kruer, Comments Plasma Physics and Controlled Fusion 2, 51 (1975).

[9] C. A. Back et al., Phys. Rev. Lett. 77, 4350 (1996).

[10] N. B. Meezan et al., Phys. Plasmas 11, 5573 (2004).

[11] S. H. Glenzer et al., Nuclear Fusion 44, S185 (2004).

[12] G. Gregori et al., Phys. Rev. Lett. 92 (2004).

[13] E. M. Epperlein and M. G. Haines, Phys. Fluids 29, 1029 (1986).

[14] E. Hollmann et al., Phys. Rev. Lett. 82, 4839 (1999).

[15] D. H. Froula et al., Phys. Rev. Lett. 93, 35001 (2004).

[16] F. Bitter, Rev. Sci. Instrum. 10, 373 (1939).

[17] B. B. Pollock et al., To Be published in RSI (2006).

[18] J. A. Fejer, Can. J. Phys. 38, 1114 (1960).

[19] D. Salzmann, Atomic Processes in Hot Plasmas (Oxford University Press, Oxford, U.K., 1998).

[20] V. Y. Bychenkov et al., Phys. Plasmas 7, 1511 (2000).

[21] S. I. Braginskii, Zh. Eksp. Teor. Fiz. 33, 459 (1957).

[22] J. D. Huba, NRL Plasma Formulary (Washington, DC, 2000).

[23] N. H. Burnett and A. A. Offenberger, J. Appl. Phys. 45, 2155 (1974). 